EGU2020-5293

https://doi.org/10.5194/egusphere-egu2020-5293

EGU General Assembly 2020

(c) Author(s) 2021. This work is distributed under

the Creative Commons Attribution 4.0 License.

\title{
Visualization of localized deformation and fluid flow in sedimentary rocks
}

\author{
Jeroen F. Van Stappen ${ }^{1,2}$, Maartje E. Houben ${ }^{1,3}$, Timotheus K.T. Wolterbeek ${ }^{1}$, Alessandro \\ Tengattini ${ }^{4,5}$, Takahiro Shinohara ${ }^{1}$, Floris S.R. Teuling ${ }^{1}$, Miao Zhang $^{1}$, Christopher J. Spiers ${ }^{1}$, and \\ Suzanne J.T. Hangx ${ }^{1}$ \\ ${ }^{1}$ Department of Earth Sciences, Utrecht University, Utrecht, The Netherlands \\ ${ }^{2}$ Now at: Department of Geology, Ghent University, Ghent, Belgium \\ ${ }^{3}$ Now at: Shell Global Solutions International BV, Amsterdam, The Netherlands \\ ${ }^{4}$ Institut Laue-Langevin, Grenoble, France \\ ${ }^{5}$ Laboratoire 3sR, Université Grenoble Alpes, Grenoble, France
}

Subsurface activities, such as energy production or geo-storage, affect the natural equilibrium of the reservoir and surrounding geological system. Fluid production from porous sandstones, for example, is often associated with reservoir compaction and induced seismicity, such as seen in the Groningen Gas Field. Production-induced stress changes lead to compaction by elastic and inelastic mechanisms. Partitioning between elastic and inelastic processes control the energy budget available for driving seismogenic events. To predict the amount of inelastic strain, it is key to identify the microscopic mechanisms controlling it. One of the current hypotheses is that microstrains are accommodated by localized compaction of inter-granular clay films. In contrast to sandstones, claystones offer potential both as source rocks for shale gas and containment for the storage of radioactive waste and $\mathrm{CO}_{2}$. It is known that fluid flow in intact and fractured claystones is slow due to pore throats below $10 \mathrm{~nm}$. However, it is unclear whether fractured shales contain a hierarchy of multi-scale highways and byways for fluid transport that is either poorly connected or more easily cross-linked and stable under in-situ conditions. Depending on how fractures change due to in-situ conditions, the shales may have a high potential as barriers in geo-storage systems, or they are of interest in relation to energy production.

This leads to two widely different research questions:

- How do sandstones compact due to changing stress conditions?

- How do fractures influence fluid flow in shales under in-situ stress conditions?

Despite the distance between these research questions, both can be addressed using in-situ imaging. We have developed a compaction cell and a fluid flow cell to perform experiments at the D50/NeXT beamline of the Institut Laue-Langevin in Grenoble, France. Here, combined X-ray and neutron imaging is possible. 
With the compaction cell, sandstone samples from the Groningen gas field were uniaxially compacted to axial stresses of $45 \mathrm{MPa}$. At different intervals, 3D neutron and X-ray computed tomography scans were taken. As such, 4D representations (3D volumetric + time) of the in-situ changes were obtained using both neutron and X-ray tomography. The X-ray imaging allows a thorough inspection of the grain-scale deformation of the sample, while the neutron imaging highlights the changes in porosity and gives an indication of the role of clay films.

With the fluid flow cell, fractured samples of the Whitby mudstone were subjected to fluid flow under different hydrostatic pressures. The flow path evolution within the sample was visualized using neutron radiography, giving an indication of the differences between fracture and matrix permeability.

In this contribution, we will show preliminary results of four experiments performed at the D50/NeXT beamline in October 2019. We will discuss the applicability of using neutron imaging to study grain-scale processes occurring in compacting sandstone, as well as for understanding the fluid pathways in clay-rich shales, with direct implications for energy production and geo-storage. 